\begin{tabular}{|c|c|}
\hline \multirow{3}{*}{ 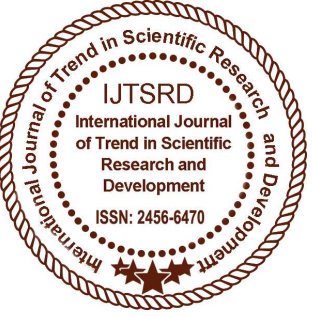 } & $\begin{array}{l}\text { International Journal of Trend in Scientific } \\
\text { Research and Development (IJTSRD) }\end{array}$ \\
\hline & International Open Access Journal \\
\hline & ISSN No: 2456 - 6470 | www.ijtsrd.com | Volume - 2 | Issue - 5 \\
\hline
\end{tabular}

\title{
Intelligent Traffic Monitoring Based on Wireless Sensor Network
}

\author{
Rajeswari. $C^{1}$, Saravanan. $\mathbf{P}^{2}$ \\ ${ }^{1}$ M. Phil. Research Scholar, ${ }^{2}$ Assistant Professor \\ D. B. Jain College (Autonomous), Thoraipakkam, Chennai, India
}

\begin{abstract}
The traffic jam is a daily-life problem in any metropolitan city. With the rise of standard of living, the number of vehicles is increasing at an exponential rate. In response to this, many researches are done in developing an intelligent traffic system. Rapid development of traffic in cities requires adaptive traffic flow control. Information on the number of vehicles in different lanes, their speed and their type is essential for adaptive management of intersections. Results are presented on real-world traffic scenes showing the tracker to be both fast and robust to vibrations, which are inevitable in traffic locations.
\end{abstract}

This study aims to test the distance measuring sensor, which can be used for both problems mentioned above. Experiments with the sensor carried out in real world conditions revealed its both strengths and weaknesses. Intelligent management of traffic flows can reduce the negative impact of congestion. In recent years, wireless networks are widely used in the road transport as they provide more cost effective options [1]. Technologies like RFID and GSM can be used in traffic control to provide cost effective solutions.

Keywords: vehicle detection, distance sensor, traffic monitor, wireless sensor network, RFID, GSM

A WSN can work during night even with poor weather conditions, when there is fog or presence of dust (pollution, volcanic ash) in the air.

WSNs are low cost and low power [2].

$>$ A WSN allows the integration of video monitoring with magnetic or power sensor. In this way, it is possible to obtain complete and integrated information (video-images and traffic volumes information) [3].

$>$ WSNs allow dynamic changes to network topology based on real needs and reports coming from sensors located along the road.

When needed, the number of cameras which control a specific area may increase to produce more detailed information. However, it may increase network workload that will be properly managed by the proposed approach.

One of the most perspective areas from the first group of applications is traffic. Traffic systems are largescale with distributed information sources by nature. Traffic system control requires different approaches than classical technology process control. Wireless sensor networks provide effective means for data collecting and transport. This makes from them an inseparable part of intelligent transport systems (ITS).

\section{INTRODUCTION}

Wireless sensor networks (WSN) represent new technology for effective solving of tasks that include distributed information sources.

$>$ A WSN can monitor and evaluate roads automatically and continuously, with little human effort.

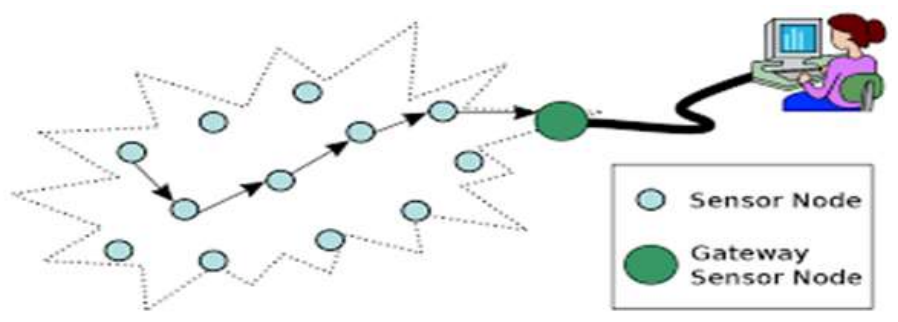


Wireless technology has propagated the use of sensor networks in many applications. Sensor networks join small sized sensors and actuators with general purpose computing components [4]. Such networks comprise of hundreds and sometimes thousands of self-functioning, low power, inexpensive wireless nodes to observe and influence the surroundings.

Traffic monitoring systems generate huge amount of data where the systems by its turn must process these useful information, especially those systems that need historical information to correctly estimate current state of traffic.

Traffic monitoring systems generally try to count, classify or estimate speed of vehicles moving on the road. It is clear that a road condition and traffic monitoring system must be economically feasible so some tuning to the system can be applied such as reducing the number of system components and reusing and utilizing existing infrastructure.

The WSN are used in multiple fields, ranging from remote environment monitoring, medical health, to home surveillance and industrial machines monitoring.

\section{OBJECTIVE:}

RFID tagging of cars is offered as an alternative to traffic data collection by inductive loops placed under the road surface. The information about the traffic collected by a network of $\mathrm{RF}$ readers is then used to regulate traffic at intersection or critical points in the city.

\section{Magnetic sensors}

In this section we describe the sensors which have been installed in the vehicle (an electric Citroën Berlingo van) and the infrastructure in order to achieve intelligent speed control. The sensors subsystems are: an RFID-based system for traffic signal detection and identification (in the infrastructure and onboard the vehicle), a differential Hall effect sensor placed in the vehicle's wheels for high accuracy speed measurement, and a differential global positioning system (DGPS) to locate the vehicle and to set the sampling frequency of our control loop

Magnetic field detection has enjoyed a significant growth thanks to several application fields: magnetic sensors can be used to detect the presence, strength or direction of magnetic fieldd is tortion caused not only by earth, but also by vehicles [4]. These sensors can measure these properties without physical contact and this is the reason why they have become so important for industrial control systems. We need to consider that magnetic sensors are never used to measure the magnetic field.

Usually they are used to evaluate parameters like speed or vehicles presence. These parameters cannot be directly calculated, but can be extracted based on magnetic field variation and distortion. Conventional sensors (temperature or pressure), can directly convert the desired parameter to a proportional voltage or current output. Using a magnetic sensor, it is necessary to measure the magnetic field variation and then process theme assured signal in order to obtain the desired output. A single axis sensor can detect vehicle presence.

\section{Radio Frequency Identification (RFID) Sensors:}

An RFID system consists in a set of emitters or tags which, periodically or upon interrogation, transmit a short digital radiofrequency message containing an identification code (unique to each tag) as well as some data stored in the tag's memory. These data can be obtained remotely by a computer equipped with an RFID reader. Besides the tag ID, which confirms the presence of the tag within the detecting range of the reader, the RFID reader measures the received signal strength (RSSI) of the RF signal, which is an indicator of the range from tag to reader.

Intelligent Speed Controller

When it comes to design a vehicle speed controller, the priority was to guarantee the car's occupants comfort. The denomination Comfort Driving is an imprecise term and its limits can be established differently according to the situation. One commonly accepted threshold in the automotive sector is found in reference [5], which fixes the maximum acceleration for comfort at $2 \mathrm{~m} / \mathrm{s} 2$.

\section{Traffic Statistics:}

A traffic monitoring system should provide the information required to alert drivers to problems, and/or, the statistics required by road engineers [6]. A list of its features may include the following:

$>$ vehicle speed, count, and lane occupancy (at a particular line across the road) surveillance over a length of road (tracking vehicles and lane- 
changing manuvres) monitoring movement through a junction

\section{METHODOLOGY:}

\section{Automatic signal control system}

Each vehicle is equipped with a RFID tag. RFID Reader Is placed at the road junction. The reader will track how many vehicles have passed through and determine the congestion volume. Reader communicates with the centralized server and thereby decides the green light duration of that path.

\section{Emergency vehicle clearance}

Emergency vehicle is also equipped with the rfid tag and when the vehicle arrives, the server detects that the vehicle is on emergency and it immediately switches the traffic light to green.

\section{Stolen vehicle detection}

The RFID reader reads the RFID tag; it compares it to the list of stolen RFIDs. If a match is found, it sends SMS to the police control room and changes the traffic light to red, so that the vehicle is made to stop at the traffic junction.

\section{Conclusion \& Enhancements}

Here We use wireless sensor network to acquire and record monitoring traffic system[8]. With automatic traffic signal control based on the traffic density in the route, the manual effort on the part of the Traffic policeman is saved. As the entire system is automated, it requires very less human intervention. With stolen vehicle detection, the signal automatically turns to red, so that the police officer can take appropriate action, if he/she is present at the junction. Also SMS will be sent so that they can prepare to catch the stolen vehicle at the next possible junctions. Emergency vehicles like ambulance, fire trucks, need to reach their destinations at the earliest. If they spend a lot of time in traffic jams, precious lives of many people may be in danger.

With emergency vehicle clearance, the traffic signal turns to green as long as the emergency vehicle is waiting in the traffic junction. The signal turns to red, only after the emergency vehicle passes through. Further enhancements can be done to the prototype by testing it with longer range RFID readers. Also GPS can be placed into the stolen vehicle detection module, so that the exact location of stolen vehicle is known. Currently, we have implemented system by considering one road of the traffic junction. It can be improved by extending to all the roads in a multi-road junction.

\section{REFERENCES:}

1. Gokulan B. P., Srinivasan D., "Distributed Geometric Fuzzy Multi-agent Urban Traffic Signal Control", IEEE Transactions on Intelligent Transportation Systems, vol.11, no.3, pp.714-727, 2010.

2. S. Deshp and, "Adaptive low-bitrate streaming over IEEE 802.15.4 low rate wireless personal area networks (LR-WPAN) based on link quality indication", International Conference on Communication and Mobile Computing, pp. 863868,2006

3. G. Pekhterverv ET. Al "Image Transmission over IEEE 802.15.4 and ZigBee Networks" IEEE International Symposium on Circuits and Systems, Vol.4, pp.3539-3543, 2005.

4. J. Lenz, "Magnetic sensors and their applications", IEEE Sensors Journal, Vol. 6, Issue 3,pp. 631649,2006

5. Bechtel C. Compendium of Executive Summaries from the Maglev System Concept Definition Final Reports. U. S. Department of Transportation; San Francisco, CA, USA: 1993. Final Rep. NO. A315823;

6. L. J. Mountain and J. B. Garner. Application of photography to traffic surveys. In The Journal of the Institution of Highway Engineers, pages 1219, November 1980.

7. S. Peachibalan. Dr. Balaji. S. P. Saravanan. Smart Grid Using Wireless Sensor Network's and Routing Protocol in International Journal of Innovative Research in Science, Engineering and Technology (IJIRSET), Vol. 6, Issue 8, Pp 1657416579, August 2017.

8. Pradeep G Pillai. P. Saravanan. A Study on Air Pollution Monitoring System using Wireless Sensor Network in International Journal of Trend in Scientific Research and Development (IJTSRD), Vol. 2, Issue 2, Pp 343-347, Jan-Feb 2018 . 